\title{
Competencia comunicacional y trabajo cooperativo en docentes
}

\author{
Betsy Janeth Bravo Cedeño \\ https://orcid.org/0000-0002-5875-7968 \\ janethbravo26@yahoo.es \\ Universidad “César Vallejo” Piura - Perú
}

Cesar Balladares Atoche cballadaresa@ucvvirtual.edu.pe https://orcid.org/0000-0001-8242-7742 Universidad “César Vallejo” Piura - Perú

Cristina Verónica Gordon Torres p700232573@ucvvirtual.edu.pe https://orcid.org/0000-0001-5192-2097 Universidad “César Vallejo” Piura - Perú

Ludia Marisol Quito Santana p7002325162@ucvvirtual.edu.pe https://orcid.org/0000-0003-4103-6467 Universidad "César Vallejo” Piura - Perú

María Patricia Unuzungo Preciado p7002323567@ucvvirtual.edu.pe https://orcid.org/0000-0001-2167-2530 Universidad "César Vallejo" Piura - Perú

\section{RESUMEN}

En la presente investigación se propuso como objetivo diseñar una estrategia de competencia comunicacional para mejorar el trabajo cooperativo en docentes. Se consideró el tipo de investigación descriptivo-propositivo, diseño transversal. La población estuvo conformada por 206 docentes y como muestra 66 docentes elegidos de forma probabilística. Se utilizó como técnica la encuesta para el recojo de información y un cuestionario como instrumento. Los datos recogidos fueron procesados e interpretados a través de la estadística descriptiva. En cuanto a la variable trabajo cooperativo se establece que los docentes consideran un $1.5 \%$ como nivel bajo; el $59.1 \%$ como medio, y un $39.4 \%$ indica que la variable en mención es alto; entre las dimensiones se evidencia una mayor problemática respecto al desarrollo de habilidades y evaluación grupal donde el $83.3 \%$ las considera como nivel medio. Se concluye que los docentes consideran que 
algunos participan de forma activa y voluntaria, demostrando así poco compromiso con la institución; concerniente al desarrollo de habilidades existen un liderazgo medio, debido a que no siempre buscan solucionar los problemas en forma conjunta, existiendo desconfianza, es necesario que reflexionen sobre las dificultades y fortalezas de las actividades desarrolladas, situaciones necesarias para lograr los objetivos institucionales.

Palabras clave: trabajo cooperativo; competencia comunicacional; trabajo en equipo; liderazgo. 


\title{
Communicational competence and cooperative work in teachers
}

\begin{abstract}
In the present investigation, the objective was to design a communicational competence strategy to improve cooperative work in teachers. The descriptive-purposeful type of research, cross-sectional design, was considered. The population consisted of 206 teachers and as a sample 66 teachers chosen probabilistically. The survey was used as a technique to collect information and a questionnaire as an instrument. The collected data were processed and interpreted through descriptive statistics. Regarding the cooperative work variable, it is established that teachers consider $1.5 \%$ as a low level; $59.1 \%$ as average, and 39.4\% indicate that the variable in mention is high; Among the dimensions, there is evidence of a greater problem regarding the development of skills and group evaluation, where $83.3 \%$ consider them as a medium level. It is concluded that teachers consider that some participate actively and voluntarily, thus showing little commitment to the institution; Regarding the development of skills, there is a medium leadership, because they do not always seek to solve problems jointly, there is mistrust, it is necessary for them to reflect on the difficulties and strengths of the activities carried out, situations necessary to achieve institutional objectives.
\end{abstract}

Keywords: cooperative work; communicational competence; teamwork; leadership.

Artículo recibido: 30 noviembre. 2021 Aceptado para publicación: 29 diciembre 2021 Correspondencia: cjanethbravo26@yahoo.es Conflictos de Interés: Ninguna que declarar 


\section{INTRODUCCIÓN}

El presente informe es producto de la investigación sobre el trabajo colaborativo, donde se abordó las dimensiones a nivel bibliográfico, en base a ellas se diseñó un instrumento que permitió recabar información de la situación en las instituciones educativas, mismas que permitieron realizar una propuesta de competencia comunicacional como respuesta a la necesidad evidente en los resultados del estudio.

La incorporación del trabajo cooperativo relacionado a la labor docente, en pleno siglo XXI, se considera como una estrategia fundamental para garantizar su desempeño con los estudiantes, considerando las circunstancias que vivimos a nivel local como mundial a consecuencia de la pandemia Covid 19; en este contexto, se han desarrollado las actividades académicas a nivel virtual en donde la cooperación entre colegas debe ser primordial para realizar un buen trabajo y sobre todo cumplir con los objetivos a nivel institucional, esto es una constante en las escuelas mexicanas como en el planeta en general (Ros, 2021).

En relación al Ecuador, los docentes, como en la mayoría de países latinoamericanos, desarrollaron sus clases de manera virtual, situación que evidencia la falta de trabajo cooperativo; por tanto, los docentes necesitan empoderarse de la situación y requieren el apoyo de los demás colegas. En el circuito 23D02C01 - Santo Domingo, se evidencia en los informes de auditoría (MINEDUC, 2017-23H00135_VL_ARG) la falta de trabajo cooperativo, los docentes presentan limitaciones al desarrollar sus actividades didácticas, ya que medianamente aplican recomendaciones para mejorar su práctica docente(p24); por ende, se recomienda se utilice estrategias de trabajo colaborativo; también es importante resaltar que a nivel institucional no se mantienen acuerdos con otros actores a fin de fortalecer el proceso educativo(p4), esto demuestra la deficiencia en las habilidades para trabajar cooperativamente a nivel de institución como a nivel de redes educativas.

El trabajo cooperativo involucra una serie de actividades estructuradas donde los maestros aprenden interactuando y colaborando entre sí, Fohlin et al. (2021) consideran que se deben desarrollar las categorías como "Capacidad, Oportunidad y Motivación". En este sentido en Suecia los docentes han manifestado un crecimiento en la categoría de capacidad para el desarrollo del trabajo cooperativo, esto implica mejorar los conocimientos (conocimientos teóricos) y habilidades (conocimientos prácticos) expresados para mejorar las habilidades de aprender junto a sus colegas; por otro lado, es 
oportuno indicar que la motivación está relacionada con el avance de las actividades planteada por los docentes.

En Ecuador Torres (2017), Riobamba, investigó sobre la "metodología cooperativa utilizando las tecnologías de la información y comunicación en el aprendizaje del idioma inglés en los estudiantes de tercer nivel de la Facultad de Ingeniería - Universidad Nacional de Chimborazo Riobamba Ecuador"; como objetivo general propuso demostrar que el aprendizaje cooperativo con aplicación de Tecnologías de la Información y Comunicación (TIC) influye en el logro de aprendizaje [...]. Se concluyó que aplicar la metodología cooperativa con la presencia de las tecnologías los estudiantes mejoró significativamente el aprendizaje del inglés, esto se pone de manifiesto en la grabación de audios por lo que desarrolla las habilidades comunicativas, además la redacción mejoró considerablemente poniendo de manifiesto las destrezas lingüísticas.

En Perú, en la Universidad César Vallejo, Collantes (2019), investigó sobre el Trabajo colaborativo y desarrollo profesional de los docentes de las instituciones educativas públicas del distrito del Cusco de la UGEL Cusco. Su objetivo fue determinar la relación existente entre trabajo colaborativo de desarrollo profesional de los docentes [...]. Se concluyó que a mejor desempeño en el trabajo colaborativo se obtuvo mejor resultado con respecto a desarrollo profesional y viceversa.

La variable competencia comunicacional se define como el conjunto estructural de capacidades, destrezas y conocimientos y que la vez está formada por aspectos lingüísticos y extralingüísticos correlacionados con las actitudes y habilidades de quién hace el rol de comunicador cuando quiere expresar sus pensamientos, emociones, definiciones, opiniones, etc. en forma de mensaje (Sánchez et al., 2011). Además, implica la habilidad de elaborar, producir y decodificar mensajes, discursos adecuados tanto en los aspectos de la oralidad; es decir, de la expresión oral como de la escritura. Estos mensajes deben cumplir con la efectividad comunicativa desarrollada en la interacción con los demás (Yuanyuan, 2019).

También se puede considerar a la competencia comunicativa como "la habilidad de la comunicación que se efectúa de manera deficiente por parte de los educandos" (Espinoza et al. 2019, p.89). Por otro lado, se deduce en este sentido se debe dar un valor importante al uso de la normas y reglas para que se desarrolle de forma correcta la práctica de la lengua y su discurso incluso los diversos alocuciones deben ser desarrollados de acuerdo 
al contexto en el que se halle el individuo, con la intención de que se cumpla el acto comunicativo (Pérez, 2017).

En relación a las dimensiones de la variable competencia comunicacional, se tiene el aporte de Canale \& Swain (1980) y, Canale (1983) que considera cuatro competencias: competencia gramatical, la misma que involucra una serie de elementos como son, reglas para el buen desarrollo de la gramática, en este sentido las reglas implica conocer "desde el nivel más elemental consistente en combinar sonidos hasta el nivel más complejo de la conversación ampliada", por ende, es muy importante conocer las reglas, porque ello implica tener un panorama general del funcionamiento de la morfología, semántica a nivel de gramática de la oración; la dimensión competencia sociolingüística planteada por Niño (2008), considera que se deben tener en cuenta los componentes en el aspecto sociocultural o formas convencionales de la palabra, en este sentido es de vital importancia el conocimiento sobre la utilización de la lengua; la dimensión competencia discursiva, se refiere a la manera en la que se combinan formas gramaticales y significados para obtener un texto hablado o escrito unificado. En este sentido, Murcia (2007), lo considera como el "conocimiento y la habilidad necesarios para producir e interpretar textos atendiendo al esquema estructural y a las convenciones lingüísticas de los diferentes géneros de discurso que usamos"; la dimensión competencia estratégica, está formada por las estrategias de comunicación verbal y no verbal, que se usan durante la interacción con el otro, además es importante tener claridad sobre algunos términos o léxico que pueden variar de un lugar a otro. (Pilleux, 2001). Frente a ello, se hace importante conocer el indicador habilidades y recursos para activar procesos mentales.

En relación al aporte de los teóricos Watzlawick et al. (1985) en su teoría de la comunicación humana basan su propuesta en un fundamento pragmático, en el cual el ser humano participa en el proceso de conocer las reglas de la comunicación ignorando en qué consisten en sí estas reglas. Según los autores la comunicación toca la sintáctica, la semántica y la pragmática. La sintáctica se enfoca en los problemas de transmitir la información centrándose en las propiedades estadísticas del lenguaje como codificación, canales, capacidad, ruido y redundancia. La pragmática se desarrolla a partir del efecto de la comunicación en la conducta y la semántica se ocupa de los significados ya que la comunicación requiere de definiciones específicas de sus términos. 
La variable de estudio trabajo cooperativo es conceptualizado por Guitert et al, (2000), como un proceso de correlación en el que intervienen los estímulos, premios, motivaciones y la ambición de lograr los objetivos, metas establecidas, la interacción entre los integrantes de equipo, las relaciones justas y armoniosas; el compromiso especial simultáneo como expresión necesaria del resultado de las metas; la gestión de habilidades comunicativas; la diversidad de información a lo específico de los mensajes, temas que permite el grupo, a cada uno de los segmentos se le retribuye labores de acuerdo a su contexto, cultura, capacidades y habilidades (p.4).

Según Johnson y Johnson (citado por Aparicio, Sepúlveda, 2019). Indica que el trabajo cooperativo está profundamente relacionado con el trabajo en equipo; por ende, se define como: conjunto de estrategias, habilidades, destrezas, procedimientos de capacitación o enseñanza en el aula para promover el avance de capacidades, destrezas y habilidades complejas. Es importante considerar el aporte de (Walss, 2015) quien indica que la habilidad de cooperación se da cuando tienen un objetivo, propósito común y trabajan en equipo para alcanzarlo. En el centro de la habilidad de la cooperación se ubica la interdependencia positiva. Esto representa que cada integrante dedica su trabajo para que todo el grupo se beneficie.

En lo que concierne a las dimensiones de trabajo cooperativo se considera la propuesta de Johnson y Johnson (citado por Aparicio, Sepúlveda, 2019). Suponen cinco dimensiones: la dimensión interdependencia positiva: se define como la dependencia entre los integrantes, la cooperación propia y de grupo para lograr el avance del trabajo y el beneficio equitativo frecuente, para lograr la Interdependencia positiva, es imprescindible, establecer un conjunto de objetivos grupales correlacionales y establecer el nivel de compromiso grupal que implique la afirmación de todas sus partes y establecer la equivalencia (Johnson et al., 1999). Con respecto a las interacciones cara a cara, según Poveda (2016) considera que cada sección debe propagar la oportunidad de acción que posibiliten acciones personales, particulares de soporte, refuerzo, apoyo, sostén, socorro y exaltación entre las secciones grupales. Interacciones con el directivo significa apoyar, animar en lugar de afrontar, buscar el bienestar de grupo, en lugar del individual y no discriminar (Velázquez, 2010).

En cuanto a la responsabilidad particular, de acuerdo con lo planteado por Johnson y Johnson (citado por Aparicio, Sepúlveda, 2019). Considera que la responsabilidad 
personal es la clave para asegurar el compromiso en conjunto en el trabajo, impidiendo de esta forma la negligencia habitual. Los estudiantes deben aprender en "conjunto habilidades, paradigmas, conocimientos, capacidades, estrategias que puedan emplear de acuerdo al contexto" (p.21). Concerniente al desarrollo de destrezas, para Escales y Pujantell, (2016) se considera que es anticipar competencias de comunicación fluida, comercializar y acompañar el rol de liderazgo, resolver problemas, habilidades, destrezas que ayuden al grupo. No siempre es un signo de validez, involucrar a un profesor en un conjunto y animarlo a colaborar ya que al profesor a veces le faltan las habilidades fundamentales del trabajo en equipo. Asimismo es fundamental en esta dimensión conocer el liderazgo que ejercen las autoridades, en este sentido, mientras el profesor más énfasis tenga y más habilidades sociales se adquieran se elevará el nivel de rendimiento de los conjuntos del bosquejo subsidiario en el aula. Referente a evaluación grupal, considerada por Leda, Cervera (2017). Indica que los elementos de un conjunto reflexionan, evalúan la información en grupo teniendo como referencia las diligencias realizadas, las metas, y los nuevos conocimientos que se traducen en el aprendizaje adquirido. Sobre este referente, se programa su plan de ajuste y mejora, se toman sus decisiones. Argumentando que, como equipo de trabajo, deberían tener como base las metas y los logros de aprendizaje. Lo antes expuesto permite considerar novedosas medidas para corregir y a cambiar el procedimiento

Teniendo en cuenta que es importante conocer la teoría en la cual se sustenta la variable trabajo cooperativo es oportuno mencionar la teoría de la definición de objetivos propuesta por Edwin Locke citada por Allowend (2017) en donde se argumenta que el centro de donde surge el motivo es el deseo de superación, de buscar y alcanzar los objetivos formulados en equipo. Donde se muestra a la persona la intensidad y las gestiones de los esfuerzos a fin de lograr los objetivos propuestos, en conclusión, se puede afirmar que esta teoría goza de una vital importancia porque permite mantener la motivación en la persona para que logre cualquier propósito; incluso se fundamente que las metas correctamente definidas ayudan a mejorar la forma de desempeño de cada uno de los integrantes. En esta perspectiva la efectividad personal permite que el individuo perciba la habilidad de resolver cada una de las tareas, actividades encomendadas, cuanto más elevado sea el nivel de eficacia individual, le permitirá aumentar el sentido de confianza del individuo y esto conlleva a un buen desempeño. Por el contrario, quienes 
presenten un nivel bajo de eficacia personal se rehúsan a esforzarse y terminan abandonando sus obligaciones, proceso muy distinto ocurre en quienes poseen un elevado grado de eficacia personal porque son ellos quienes demuestran mejor desenvolvimiento en sus cargos y se esfuerzan con mayor tenacidad. (Aldana \& Piña, 2017).

Por lo descrito anteriormente surgió la necesidad formular la siguiente pregunta de investigación: ¿Cómo la competencia comunicacional mejora el trabajo cooperativo en docentes, circuito 23D02C01 - Santo Domingo - 2021?, como objetivo general se planteó diseñar una estrategia de competencia comunicacional para mejorar el trabajo cooperativo en docentes, circuito 23D02C01 - Santo Domingo - 2021. Se plantearon como objetivos específicos: diagnosticar el nivel de trabajo cooperativo en docentes, circuito 23D02C01 - Santo Domingo - 2021; analizar las teorías que sustentan la estrategia de competencia comunicacional; elaborar una estrategia de competencia comunicacional para mejorar el trabajo cooperativo en docentes, circuito 23D02C01 - Santo Domingo - 2021; validar estrategia de competencia comunicacional para mejorar el trabajo cooperativo en docentes, circuito 23D02C01 - Santo Domingo - 2021. Por otro lado, de acuerdo a la característica del tipo de investigación no se consideró hipótesis.

\section{ESTRATEGIAS METODOLÓGICAS O MATERIALES Y MÉTODOS}

La presente indagación se considera básica, según Ary et al (1989), considerando que "busca mencionar las cualidades o características preestablecidas de un fenómeno o grupo de personas". De acuerdo a su temporalidad se la considera de tipo transversal. Considerando a Lakatos (1998), la investigación fue propositiva la misma que estuvo orientada a responder de manera positiva a los problemas diagnosticados. El diseño descriptivo, concordando con Sánchez \& Reyes (1998), no se manipuló ninguna variable, se observó los fenómenos desde su propia naturaleza (p. 77).

Como instrumento se utilizó la encuesta, la misma que sirvió para recopilar la información sobre trabajo cooperativo. Esta fue diseñada de acuerdo a los fines investigativos en concordancia con Latorre et al (1996). Para el recojo de información se consideró el cuestionario, que a decir de Pino (2007) reside en "un acumulado de preguntas" que están redactadas en forma coherente en función de la operacionalización de la variable. Para medir la fiabilidad de la consistencia interna de los ítems que la conforman, se utilizó el alfa de Cronbach, recurso que describe la fiabilidad del instrumento, se utiliza expresiones como: coeficientes de precisión, estabilidad, 
equivalencia, homogeneidad, o consistencia interna, cuyo denominador común se expresan como coeficientes de correlación (Hernández, Mendoza, 2018).

Se consideró una población de 206 docentes pertenecientes al circuito 23D02C01. En consecuencia, Latorre et al (1996) consideran a la población como un conjunto finito o infinito de elementos con características comunes que forman parte del espacio territorial y que presentan un conjunto de particularidades en un contexto específico. Teniendo en cuenta que la muestra es un "subconjunto de la población y al mismo tiempo debe ser representativa debido a que se consideran fielmente las características de ella" Carrasco, 2009, p.92). Se consideró la muestra de 66 docentes, ver anexo 6. Se utilizó el muestreo probabilístico debido a que "todos los elementos tienen la posibilidad de ser elegidos" (Carrasco, 2009, p. 241). Para determinar los docentes que eran parte de la muestra de estudio se empleó el muestreo por conglomerados mismo que consistió en seleccionar aleatoriamente las instituciones educativas del circuito.

\section{RESULTADOS Y DISCUSIÓN}

Dado los objetivos planteados se consideró presentar los resultados en función al objetivo específico 1: diagnosticar el nivel de trabajo cooperativo en docentes, circuito 23D02C01 - Santo Domingo - 2021.

Tabla 1 Variable trabajo cooperativo en docentes

\begin{tabular}{ccc}
\hline Nivel & Frecuencia & Porcentaje \\
\hline Bajo & 1 & 1,5 \\
Medio & 39 & 59,1 \\
Alto & 26 & 39,4 \\
\hline Total & $\mathbf{6 6}$ & $\mathbf{1 0 0 , 0}$ \\
\hline
\end{tabular}

Nota. En la Tabla 1 se hace referencia a los datos recogidos a través del instrumento aplicado los docentes, circuito 23D02C01 - Santo Domingo - 2021.

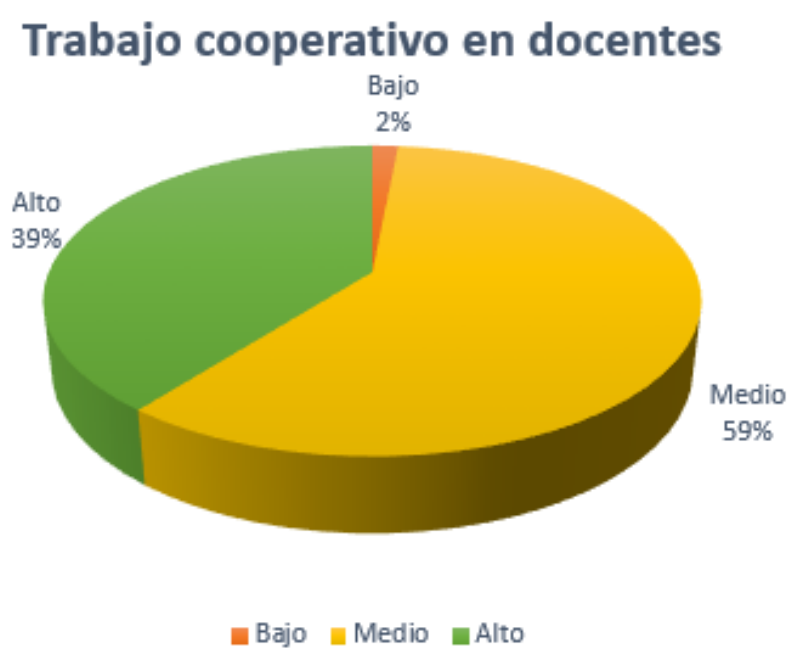


Considerando el objetivo específico "diagnosticar el nivel de trabajo cooperativo en docentes, circuito 23D02C01 - Santo Domingo - 2021" se fundamenta en el aporte teórico de Walss, (2015) en donde indica que la habilidad de cooperación se da cuando tienen un objetivo, propósito común y trabajan en equipo para alcanzarlo. Guitert \& Giménez, (2000) sostienen que el trabajo cooperativo comprende la intervención de los estímulos, premios, motivaciones y la ambición de lograr los objetivos, metas establecidas de carácter institucional. En este sentido la Tabla 1 nos indica que un 1.5\% de los maestros consideran a la variable en mención que su desempeño es de nivel bajo; además del $59.1 \%$ sostiene que es de nivel medio, y un $39.4 \%$ indica que la variable en mención es alto. Esto nos permite coincidir con Tarraga, (2018) que considera en su investigación el trabajo colaborativo en un $28,9 \%$ es regular en tal sentido se debe mejorar.

De acuerdo a estos resultados se puede inferir que la efectividad del trabajo permite que las personas desarrollen la habilidad de resolver cada una de las tareas, actividades encargadas, con responsabilidad, además del compromiso institucional que debe ponerse de manifiesto en cada de las acciones, es por ello la importancia de tener un aceptado liderazgo con el manejo de habilidades para tratar de entender la problemática en cada participante, el mismo que permitirá aumentar el sentido de confianza del individuo y esto conllevará a un buen desempeño dentro de la institución.

De las dimensiones estudiadas referente a la variable trabajo cooperativo, se han considerado las dos que evidencian una mayor problemática en base a los resultados, mismas que se detallan a continuación.

\section{Tabla 2}

Dimensión desarrollo de habilidades en el trabajo cooperativo

\begin{tabular}{ccc}
\hline Nivel & Frecuencia & Porcentaje \\
\hline Bajo & 2 & 3,0 \\
Medio & 55 & 83,3 \\
Alto & 9 & 13,6 \\
\hline Total & $\mathbf{6 6}$ & $\mathbf{1 0 0 , 0}$ \\
\hline
\end{tabular}

Nota. En la Tabla 2 se hace referencia a los datos recogidos a través del instrumento aplicado los docentes, circuito 23D02C01 - Santo Domingo - 2021 


\section{Desarrollo de habilidades en el trabajo cooperativo}

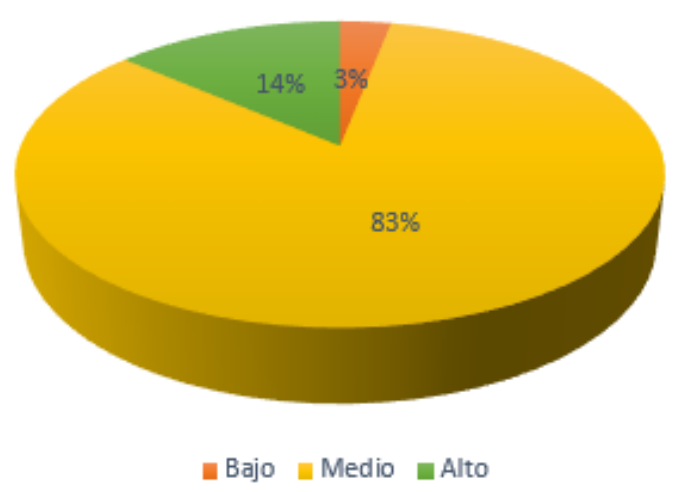

Según los datos recogidos tenemos que para la dimensión desarrollo de habilidades, la Tabla 2 encontramos que el 3.0\% de los maestros encuestados califica a esta dimensión como nivel bajo; el $83.3 \%$ consideran que es medio, y un $13.6 \%$ sostiene que la dimensión es alto. Es evidente que el desarrollo de habilidades de los equipos de trabajo son pocos desarrollados por sus integrantes quienes deberían plantear alternativas de solución en forma conjunta, desarrollando confianza y sobre todo comprometiéndose al cumplimiento de los objetivos institucionales.

Escales, Pujantell, (2016) consideran que en esta dimensión se debe desarrollar destrezas como la comunicación fluida, liderazgo, la capacidad para resolver problemas, además de plantear habilidades, destrezas que ayuden al desenvolvimiento del grupo. Los resultados expuestos nos permiten coincidir con el planteamiento de Collantes (2019), en el cual establece que la aplicación de destrezas mejoran el desempeño en el trabajo colaborativo, obteniendo mejores resultados al momento de trabajar con la participación de los miembros de la institución; además de López et al. (2021) en donde establece que la confianza, creatividad y el trabajo ayuda a lograr los objetivos establecidos a inicio de año en las instituciones.

Estos resultados nos permiten deducir que la dimensión desarrollo de destrezas en lo que corresponde al trabajo en equipo es fundamental que sean liderados por la totalidad de sus integrantes y que busquen plantear alternativas de solución en forma conjunta, además de tener en consideración la labor del líder que debe actuar de acuerdo a características democráticas y que brinde el nivel de empatía, y sobre todo brindar la confianza para poder cumplir con los objetivos institucionales. Esto es importante debido a que los resultados muestran que existen habilidades y destrezas que coadyuvan al cumplimiento 
de metas y sobre todo lo más importante es la forma como se desenvuelven los integrantes, además la labor que desempeñan los líderes en donde se evidencia participación de forma democrática y sobre todo compromiso de trabajar en bien de la institución a la cual forman parte, en este sentido se consiguen las metas y objetivos planteados.

\section{Tabla 3}

Dimensión evaluación grupal en el trabajo cooperativo

\begin{tabular}{ccc}
\hline Nivel & Frecuencia & Porcentaje \\
\hline Bajo & 2 & 3,0 \\
Medio & 55 & 83,3 \\
Alto & 9 & 13,6 \\
\hline Total & $\mathbf{6 6}$ & $\mathbf{1 0 0 , 0}$ \\
\hline
\end{tabular}

$\overline{\text { Nota. En la Tabla } 3 \text { se hace referencia a los datos recogidos a través del instrumento }}$ aplicado los docentes, circuito 23D02C01 - Santo Domingo - 2021.

\section{Evaluación grupal en el trabajo cooperativo}

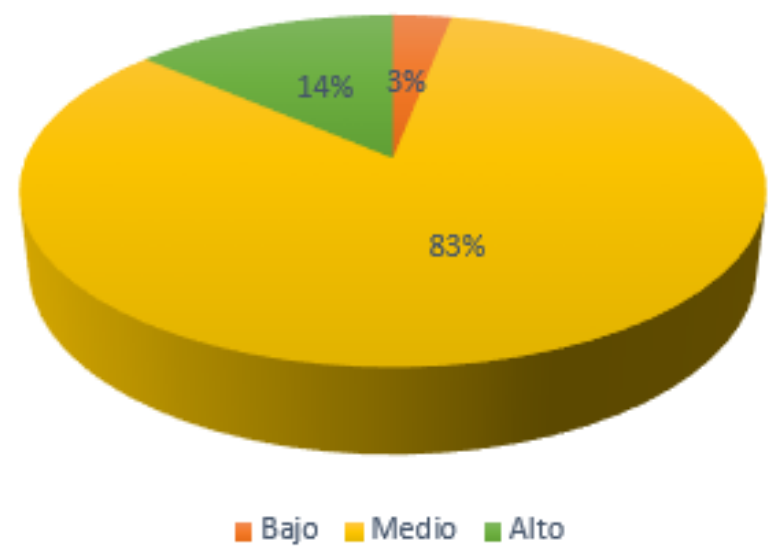

Para la dimensión evaluación grupal tenemos que la Tabla 3 en donde encontramos que el 3.0\% de los maestros encuestados considera que la dimensión en mención es de nivel bajo; además del $83.3 \%$ consideran que es medio, asimismo un $13.6 \%$ sostiene que la dimensión es alta. Los resultados evidencian que en lo grupos de trabajo muy poco reflexionan sobre su participación en el desarrollo de las actividades planteadas, es por 
ello, que frente a las dificultades necesitan restructurar las estrategias para lograr el cumplimiento de los objetivos y metas de la institución.

Leda, Cervera (2017), sostienen que los elementos a evaluar en toda acción deben partir por reflexionar la práctica, además de autoevaluarse en grupo teniendo como referencia las diligencias realizadas, las metas, y los nuevos conocimientos que se traducen en los logros conseguidos. Estos resultados nos permiten coincidir con la investigación de Guerrero \& Sánchez (2019), en el cual establece la importancia de planificar, gestionar y evaluar el aprendizaje.

De acuerdo al acápite anterior se puede inferir que los maestros organizados en grupos de trabajo reflexionan sobre su participación en el desarrollo de las actividades planteadas, esta proceso de análisis de sus actividades les permite reestructurar las dificultades identificadas y plantear algunas estrategias para lograr el cumplimiento de los objetivos y metas de la institución, es por ello que se considera importante este proceso de reflexión, teniendo en cuenta los reajustes en el trabajo.

\section{CONCLUSIÓN O CONSIDERACIONES FINALES}

Concerniente a la variable trabajo cooperativo un $59.1 \%$ sostiene que se encuentra en un nivel medio, y un $39.4 \%$ indica que se ubica en el nivel alto, estos datos nos permiten inferir que algunas veces se reciben beneficios equitativos, hay escaza participación de forma activa y voluntaria, deficiente compromiso para buscar soluciones, los docentes asumen su trabajo con poca responsabilidad demostrando un compromiso medio con la institución, además del limitado trabajo en equipo para lograr los objetivos institucionales, generando un clima de desconfianza entre los miembros, los resultados reflejan una escaza reflexión entre sus miembros, sobre sus compromisos, análisis de las dificultades y fortalezas de las actividades desarrolladas en la institución.

A los rectores de las instituciones educativas desarrollar talleres y capacitaciones continuas sobre trabajo en equipo y empoderamiento, relaciones interpersonales, habilidades blandas, para poder adquirir estrategias de manejo de emociones, solución de conflictos, y toma de decisiones, mismas que fomentan el cumplimiento de responsabilidades demostrando eficiencia y eficacia.

Se considere la aplicación de la propuesta a fin de aportar al crecimiento institucional, ya que esta ha sido diseñada considerando las teorías pertinentes y previa validación de 
expertos, permitiendo mejorar el trabajo cooperativo en docentes, el mismo que garantiza lograr los objetivos estratégicos.

\section{LISTA DE REFERENCIAS}

Aldana, J., \& Piña, J. (2017). Quality of service provided to the client by gym instructors. Interdisciplinary Arbitrated Review Koinonía, 2 (3), 172-197. http://fundacionkoinonia.com.ve/ojs/index.php/revistakoinonia/article/view/59/4 6

Allowend, T. (2017). Group Personality Composition and Group Effectiveness: An Integrative Review of Empirical Research. https://journals.sagepub.com/doi/10.1177/1046496404268538

Aparicio, C., Sepúlveda, F. (2019). Trabajo colaborativo docente: nuevas perspectivas para el desarrollo docente. Int. Investig. Cienc. Soc, 120 - 122.

Ary, D.; Jacobs, L.; Razavieh, A. (1989). Introducción a la investigación pedagógica. Segunda edición. México. McGRAW-HILL

Canale, M., Swain, M (1980). Theoretical bases of communicative approaches to second lenguaje teaching and testing. Applied Lingustics. 1(1), 29-31.

Carrasco, S. (2009). Metodología de la investigación científica. Lima, Perú: San Marcos.

Collantes, A. (2019). Trabajo colaborativo y desarrollo profesional de los docentes de las instituciones educativas públicas del distrito del Cusco de la UGEL Cusco. (Tesis doctoral, Universidad César Vallejo). https://repositorio.ucv.edu.pe/bitstream/handle/20.500.12692/44065/Collantes_Q A-SD.pdf? sequence $=1$ \&isAllowed $=\mathrm{y}$

Escales, R., y Pujantell, M. (2016). Habilidades sociales. Madrid: Macmillan Iberia, S.A. Espinoza, E., Rivas, H., Lema, R., Reyes, G. y Calvas, M. (2019). Formación de competencias comunicativas. Carrera de educación básica. UTMach. Ecuador. Espacio, 40(41), 21. http://www.revistaespacios.com/a19v40n41/a19v40n41p21.pdf

Fohlin, L., Sedem M. Allodi M. (2021). Teachers' Experiences of Facilitators and Barriers to Implement Theme-Based Cooperative Learning in a Swedish Context. Front. Educ. 6:663846. Doi: 10.3389/feduc.2021.663846 
Guerrero, M. y Sánchez M. (2019). Aprendizaje colaborativo en el sistema de educación superior ecuatoriano. Artículo científico revista Redalyc. https://www.redalyc.org/jatsRepo/280/28059953011/28059953011.pdf

Guitert, R., Giménez, M. (2000). El trabajo cooperativo en entornos virtuales de aprendizaje. Barcelona: Gedisa.

Hernández, R., Mendoza, C. (2018). Metodología de la investigación. Las rutas cuantitativa, cualitativa y mixta. México: McGraw-Hill/Interamericana Editores.

Johnson, D. W., Johnson, R. T., \& Holubec, E. J. (1999). El aprendizaje cooperativo en el aula. Buenos Aires: Editorial Paidós.

Lakatos, I. (1998). La metodología de los programas de investigación científica. Madrid: Alianza Universidad.

Latorre, A.; Delio del Rincón, I.; Arnal, J. (1996). Bases metodológicas de la investigación educativa. Barcelona: GR92.

Leda, S.; Cervera, O. (2017). Trabajo colaborativo como estrategia didáctica para el desarrollo del pensamiento crítico. (Tesis de doctoral, Universidad de la Costa Cuc,

Colombia).

https://repositorio.cuc.edu.co/bitstream/handle/11323/111/32853821-

\%2022468706.pdf?sequence $=1 \&$ isAllowed=y

López, I., Llobet, G., Bonastra, Q., \& Jové, G. (2021). Investigando colaborativamente en el doctorado: Bondades y desafíos a partir de un estudio de caso. Archivos Analíticos de Políticas Educativas, 29(58). https://doi.org/10.14507/epaa.29.5587

Murcia, C. (2007). Rethinking the Role of Communicative Competence in Language Teaching. En Eva Alcón Soler y Maria Pilar Safont Jordà (Eds.), Intercultural Language Use and Language Learning, 41-57. Dordrecht: Springer. https://www.upf.edu/web/ecodal/glosario-competencia-discursiva

Niño, V. (2008). Competencias en la comunicación. Hacia las prácticas del discurso. Bogotá. Ecoe Ediciones.

Pérez, Y. (2017). La competencia comunicativa del docente en el ámbito escolar. Transformación, $13(3)$, 394-405. http://scielo.sld.cu/scielo.php?script=sci_arttext\&pid=S207729552017000300009 
Pilleux, M. (2001). Competencia comunicativa y análisis del discurso. https://scielo.conicyt.cl/scielo.php?script=sci_arttext\&pid=S007117132001003600010

Pino, R. (2007). Metodología de la investigación. Lima, Perú: Editorial San Marcos.

Poveda, P. (2016). Implicaciones del aprendizaje de tipo cooperativo en las relaciones interpersonales y el rendimiento académico. España: Universidad de Alicante.

Ros, J. (2021). Innovations in the educational field: ict and cooperative work in primary education. https://dialnet.unirioja.es/servlet/articulo?codigo $=7767302$

Sánchez, H., Reyes, C. (1998). Metodología y diseños en la investigación científica. Lima: Perú. Editorial Mantaro.

Sánchez, M., Betancourt, C., y Reyes, C. (2011). El efecto de rebote y la expresión oral en inglés. Ciencias Holguín, 16(4),1-11.

Tarraga, L. (2018). Trabajo cooperativo y las prácticas preprofesionales en los estudiantes del VIII ciclo de la Especialidad de Educación Inicial de la Universidad Nacional de Educación Enrique Guzmán y Valle, 2017. https://www.lareferencia.info/vufind/Record/PE_19321428a1ed68eb1e3e1ae595 $4621 \mathrm{e} 6$

Torres, J., García, J., Herrero, E. (2020). Contributions of technology to cooperative work for university innovation with Design Thinking [Aportaciones de la tecnología al trabajo cooperativo para la innovación universitaria con Design Thinking]. https://doi.org/10.12795/pixelbit.74554

Velázquez. J. (2010). Aprendizaje cooperativo. Barcelona: Inde.

Walss, M. (2015). El trabajo colaborativo: una herramienta de los docentes y para los docentes. Buenos Aires: Campus Laguna.

Yuanyuan, C. (2019). Enhancing EFL Students' English Competency Through Drama: A Case Study in a Primary School in China. English Language Teaching, 12(7), 68-74. 